\title{
Impact of age on morbidity and outcome of concurrent radiochemotherapy in high-risk FIGO stage I to IVA carcinoma of the uterine cervix following laparoscopic surgery
}

\author{
Thea Laurentius • Annelore Altendorf-Hofmann • \\ Oumar Camara $\cdot$ Ingo B. Runnebaum · \\ Thomas G. Wendt
}

Received: 17 March 2010 / Accepted: 23 April 2010 / Published online: 15 May 2010

(C) The Author(s) 2010. This article is published with open access at Springerlink.com

\begin{abstract}
Purpose To evaluate the impact of age on treatmentrelated acute morbidity, on modifications of drug doses and radiotherapy and on disease-free and overall survival in non-elderly and elderly with high-risk cervical cancer treated with concurrent radiochemotherapy following laparoscopic surgery.

Methods One hundred and two patients with high-risk FIGO I - IVA cervical cancer (77 non-elderly [ $<60$ years] and 25 elderly [ $\geq 60$ years]) were treated by radical hysterectomy with lymphadenectomy $(n=91)$ and postoperative radiochemotherapy or radical radiochemotherapy alone after laparoscopic lymph node dissection $(n=11)$ patients received five to six cycles of cis-platin, $40 \mathrm{mg} / \mathrm{sqm}$ weekly, given concurrently to pelvic radiotherapy of 45-50.4 Gy in 5-6 weeks. Paraaortic radiotherapy with 45 Gy was performed when laparoscopic staging revealed paraaortic node metastases. Acute morbidity was prospectively scored weekly. Toxicity-related protocol violations (treatment breaks or dose reduction) were related with age, 5-year overall survival (OS) and progression-free survival (PFS) rates.

Results Clinical stages and histologic subtypes were equally distributed. Grade 3/4 leukopenia, anemia, diarrhea and nausea occurred more frequently in the elderly. 16 (22\%) non-elderly and $6(25 \%)$ elderly needed a modification of drug dose. 10/77 (13\%) non-elderly patients and 11/25 $(44 \%)$ of the elderly needed an unscheduled treatment break $(p=0.002)$. OS and PFS were not different between
\end{abstract}

T. Laurentius · A. Altendorf-Hofmann · O. Camara ·

I. B. Runnebaum · T. G. Wendt $(\square)$

Friedrich-Schiller-University Jena, Jena, Germany

e-mail: thomas.wendt@med.uni-jena.de age groups. The 5-year OS rate was $47 \pm 6 \%$ for non-elderly patients and $45 \pm 10 \%$ for the elderly. Patients with/without treatment breaks had a 5-year OS rate of $39 \pm 11 \%$ / $48 \pm 6 \%$. The 5 -year PFS rate is $49 \pm 6 \%$ for non-elderly patients and $47 \pm 11 \%$ for the elderly. Patients with/ without treatment breaks had a 5-year PFS probability of $50 \pm 12 \% / 48 \pm 6 \%$. FIGO stage retains its prognostic significance irrespective of age.

Conclusions Concurrent radiochemotherapy caused slightly higher acute toxicity with increasing age. Adjustment of treatment intensity was more frequent in the elderly but did not result in detrimental outcome figures.

Keywords Cervical cancer - Carcinoma of uterine cervix Radiochemotherapy $\cdot$ Chemoradiation · Elderly ·

Non-elderly $\cdot$ Morbidity $\cdot$ Laparoscopic surgery

\section{Introduction}

A combination of surgery, radiotherapy and chemotherapy has become a mainstay treatment for high-risk invasive carcinoma of the uterine cervix, since increased survival after multimodal therapy compared to surgery or radiotherapy alone has been reported in randomized studies in the late 90s (Morris et al. 1999; Rose et al. 1999; Green et al. 2001). Since the recommendation of The National Cancer Institute of the United States of America to consider cisplatinum containing chemotherapy to be given in addition to radiotherapy in 1999 (McNeil 1999), new strategies and surgical techniques have been introduced for dissection of pelvic and paraaortic lymph nodes which result in an improved pathologic staging (Altgassen et al. 2000; Köhler et al. 2004). Likewise, new radiation technologies have recently emerged such as three-dimensional conformal 
radiotherapy and intensity modulated radiotherapy, both allowing for reduced exposure of normal tissues (Ahamad et al. 2005; Vandecasteele et al. 2009).

Simultaneous radiochemotherapy has shown to increase hematologic and gastrointestinal toxicity that may compromise patients' compliance in patients receiving adjuvant radiochemotherapy due to risk factors (Peters et al. 2000). The new surgical and radiotherapeutic strategies and techniques however also harbor a specific profile of adverse effects which may contribute specifically to modified treatment-related morbidity. Systematic lymph node dissection will result in increased subacute and late toxicity like infection, lymphoceles and chronic lymph edema of the lower extremities particularly when more than 25 nodes have been removed (Füller et al. 2008).

At the Jena University Hospital in the late 1990s, systematic pelvic and paraaortic laparoscopic lymph node dissection and radiochemotherapy irrespective of age has been adopted for high-risk invasive cancer of the uterine cervix as a routine method. A significant proportion of patients are elderly. Little has been reported on toxicity and morbidity of multimodal treatment concepts including invasive laparoscopic staging with special attention to age. We performed a retrospective analysis of elderly defined as 60 years and over at treatment start and non-elderly patients diagnosed with high-risk carcinoma of the uterine cervix with respect of treatment intensity, acute morbidity and treatment outcome. To ascertain as much treatment homogeneity as possible only patients were included with their surgery and radiochemotherapy carried out at the Departments of Gynecology and Radiation Oncology of the Jena University Hospital.

\section{Patients and methods}

From the University Hospital Tumor Registry and from the Local Area Network Treatment and Information System (LANTIS) of the Dept. Radiation Oncology a list of all patients treated for cervical carcinoma at the University Hospital between 1/1999 and 12/2007 has been compiled. Consecutively treated women were included in this analysis if they have received postoperative radiochemotherapy due to at least one risk factor or if they were considered inoperable due to local primary extension and/or extensive paraaortic lymph node metastases or comorbidity. Risk factors in their postoperative pathology report were considered stage pT1b2 or higher, histologically proven lymph node metastases, lymphatic vessel involvement and close margin resection, deep stromal invasion, adenosquamous cell carcinoma or adenocarcinoma and parametrial involvement. Patients not eligible for radical surgery of the primary or with a pelvic recurrence after surgery alone were treated by radical external beam radiotherapy and brachytherapy and concurrently given chemotherapy. None had preoperative radiotherapy or radiochemotherapy. When staging procedures revealed distant metastases patients were excluded from this analysis. One hundred and two patients met these criteria and form the basis for this retrospective analysis.

\section{Surgery}

For surgical treatment of the cervical carcinoma, radical hysterectomy was performed as laparoscopic assisted radical vaginal hysterectomy (LARVH) until 12/2005, later as vaginal assisted laparoscopic radical hysterectomy (VALRH). All patients received systematic laparoscopic pelvic and paraaortic lymph node dissection and histopathological staging.

\section{Radiotherapy}

All patients received external radiation treatment. Irradiated volume was confined to the pelvis (encompassing the presacral and pelvic lymph nodes, the upper vagina and the cervical tumor if surgery has not been performed) only when paraaortic nodes specimen revealed no metastasic deposits at microscopic examination. In patients with proven paraaortic lymph node metastases, the irradiated volume included the pelvis and paraaortic nodes up to the level Th 12 treated in a sequential manner. When paraaortic irradiation was indicated, it was started after a break of 1-3 weeks after completion of pelvic radiotherapy.

Total external beam radiation dose of the pelvis was $45-50.4$ Gy $(5 \times 1.8 \mathrm{~Gy} /$ week prescribed to the $100 \%$ Isodose and the $95 \%$ Isodose encompassing the aforementioned volume) utilizing computerized tomography-based treatment planning. During the initial period, a conformal four field box was used. Recently, intensity modulated radiotherapy using a step and shoot technology has become an institutional standard technique to treat the pelvis. In cases of marginal tumor resection, patients received an additional external beam boost dose confined to iliac nodes/lateral pelvic wall to $50.4 \mathrm{~Gy}$. When indicated the paraaortic nodes were treated by external beam conformal four field box technique with a total dose of $45 \mathrm{~Gy}(5 \times 1.8 \mathrm{~Gy} /$ week $)$. All irradiations were performed with 15-MV photons.

External beam radiation was followed by intracavitary brachytherapy by high-dose rate remote afterloading technique using 192- Iridium as radioactive source or an alternative form of boost. After hysterectomy, the boost dose was given with two or three brachytherapy fractions 5-7 Gy each at $5 \mathrm{~mm}$ tissue depth to the vaginal vault spaced by 1 week.

In patients retaining their uterus, a total dose of $35-40 \mathrm{~Gy}$ to point A (International Commission on Radiation Units and Measurements Report 62) was prescribed using five to 
seven (median 6) insertions of a uterine applicator using high-dose remote afterloading with 192-Iridium as radioactive source. None had interstitial implants. In cases where a cervical insertion was not successful, the boost was given by reduced external four field box technique.

\section{Concomitant chemotherapy}

\section{Pretreatment investigations}

Patients were eligible for cis-platinum containing treatment protocol if the renal function measured by the tubular secretion of 99 m-Tc-mercaptoacetyltriglycin (MAG-3) was normal (clearance minimum: $188 \mathrm{ml} / \mathrm{min} / 1.73 \mathrm{sqm}$ body surface area $[\mathrm{BSA}])$ and audiometry did not reveal severe hypacusis (defined as a loss of $\geq 25 \mathrm{~dB}$ or hypacusis at $8,000 \mathrm{~Hz}$ or greater). In patients with reduced MAG-3 clearance or with severe hypacusis diagnosed before start of therapy the dose of cis-platinum was reduced appropriately or was substituted by vinorelbine. Eligibility criteria included normal blood cell count including absolute neutrophil count of $2 \mathrm{G} / 1$ or more, normal hemoglobin, normal serum liver enzymes and electrolytes as well as normal renal function parameters including creatinine clearance, and an ECOG performance status of 0-2.

\section{Treatment schedules}

All radiochemotherapy courses have been administered on an inpatient basis. Chemotherapy was given simultaneously external beam radiotherapy of the pelvis only. During paraaortic radiotherapy, chemotherapy has not been given. Basically two types of chemotherapy protocols have been applied in this cohort. At the beginning of the period, reported five patients received a combination of cis-platinum with 5-fluorouracil (5-FU) according to the department's guidelines. These patients received 5-fluorouracil, $1.5 \mathrm{~g}$ absolute dose $/ 24 \mathrm{~h}$ over $96 \mathrm{~h}$ as continuous infusion starting on days 1 and 29 and cis-platinum, $20 \mathrm{mg} / \mathrm{sqm}$ BSA as a $2 \mathrm{~h}$ infusion on days $1-5$ and $29-33$ of pelvic radiotherapy. The number of cycles scheduled was 2 . All subsequent patients were eligible to a protocol scheduling cis-platinum once a week at a dose of $40 \mathrm{mg} / \mathrm{sqm}$ BSA given as a $2 \mathrm{~h}$ i.v. infusion. Hydration with 2 liters normotone saline i.v. and furosemide $24 \mathrm{~h}$ before and $24 \mathrm{~h}$ after end of cis-platinum administration was obligatory. Patients with impaired renal function and/or mild hypacusis or other ineligibilities for full dose cis-platinum started their therapy with cis-platinum, 30 or $20 \mathrm{mg} / \mathrm{sqm}$ BSA weekly. In patients with contraindications against cis-platinum vinorelbine $20 \mathrm{mg} / \mathrm{sqm}$ BSA was given instead orally once a week. The scheduled number of cycles was 5 or 6 depending on the total irradiation time elapsed.
All patients had routine blood cell counts including neutrophils once a week and additional counts when indicated. Adverse effects were prospectively documented on a daily basis and their severity was classified according to the common toxicity criteria (NIH 1998). The highest grade of every type of adverse effects was used for this analysis. Drug dose delivered at each cycle was modified when during radiochemotherapy one of the following toxicities grade 2 or higher occurred: leukocyte count, anemia, emesis, thrombopenia or diarrhea. When anemia grade 3 occurred (transfusion indicated) administration of cis-platinum was terminated.

Follow-up and statistics

All women have been followed up at regularly intervals mainly at the Department of Gynecology and Obstetrics of the University Hospital until 12/2009 or at the date of their death, except for two patients. These two were younger than 60 years returned to their home country outside Germany after therapy has been completed. Data of local control status, distant metastasis status and overall survival status were supplied by the Tumor Registry. This analysis was focused on the comparison between age groups of nonelderly ( $<60$ years) and elderly ( $\geq 60$ years) patients with respect of acute toxicity, progression free and overall survival. Survival curves were estimated by the Kaplan-Meier method. Comparison of survival probabilities were analyzed using the log-rank test. All statistical tests were two sided and carried out at the 5\% level of significance.

\section{Results}

Table 1 provides patients' demographic and treatment characteristics. Median age at start of treatment was 47.7 (23-84) years, 25 women of them being 60 years or older. Median follow-up time for all patients was 34.5 (4-109) months and for surviving patients 59 (27-109) months. One non-elderly patient died from unknown cause of death. One elderly patient died from breast cancer.

Clinical stage of disease revealed an equal distribution across the age cohorts $(p=0.936)$. Likewise the distribution of the histological tumor type (squamous carcinoma, adenocarcinoma, adenosquamous carcinoma) is equal across both age groups $(p=0.552)$. The frequence of node positive tumors was high in both groups $(58 \%$ in the younger group vs. $48 \%$ in the elderly; Table 1).

Seven non-elderly and 4 elderly had no surgical removal of the primary. Twenty-six non-elderly and 9 elderly patients did not receive brachytherapy, either because of refusal or due to complicating medical conditions that precluded applicator placement. 
Table 1 Patients' demographic and treatment characteristics $(n=102)$

\begin{tabular}{|c|c|c|}
\hline & $\begin{array}{l}\text { Non-elderly } \\
\text { patients } \\
(n=77)\end{array}$ & $\begin{array}{l}\text { Elderly patients } \\
(n=25)\end{array}$ \\
\hline Median age (range) & $44(23-59)$ & $67(60-84)$ \\
\hline Tumor stage (FIGO) & $n(\%)$ & $n(\%)$ \\
\hline I & $30(39 \%)$ & $10(40 \%)$ \\
\hline II & $24(31 \%)$ & $8(32 \%)$ \\
\hline III & $13(17 \%)$ & $3(12 \%)$ \\
\hline IV & $10(13 \%)$ & $4(16 \%)$ \\
\hline Node negative & $32(42 \%)$ & $13(52 \%)$ \\
\hline Node positive & $45(58 \%)$ & $12(48 \%)$ \\
\hline \multicolumn{3}{|l|}{ Histology } \\
\hline Squamous & $63(82 \%)$ & $20(80 \%)$ \\
\hline Adenocarcinoma & $7(9 \%)$ & $1(4 \%)$ \\
\hline $\begin{array}{l}\text { Adenosquamous } \\
\text { carcinoma }\end{array}$ & $2(3 \%)$ & $2(8 \%)$ \\
\hline Other & $5(6 \%)$ & $2(8 \%)$ \\
\hline \multicolumn{3}{|l|}{ Surgery: hysterectomy } \\
\hline Yes & $70(91 \%)$ & $21(84 \%)$ \\
\hline No & $7(9 \%)$ & $4(16 \%)$ \\
\hline \multicolumn{3}{|l|}{ Chemotherapy regimen } \\
\hline \multicolumn{3}{|l|}{ Dosage $^{\mathrm{a}}$} \\
\hline Cis-platinum $40 \mathrm{mg}$ & 69 & 21 \\
\hline Cis-platinum $30 \mathrm{mg}$ & 1 & 0 \\
\hline Cis-platinum $20 \mathrm{mg}$ & 1 & 1 \\
\hline $\begin{array}{l}\text { Cis-platinum } 20 \mathrm{mg}+ \\
\text { 5-FU } 600 \mathrm{mg}\end{array}$ & 2 & 0 \\
\hline $\begin{array}{l}\text { Cis-platinum } 20 \mathrm{mg}+ \\
\text { 5-FU } 1,000 \mathrm{mg}\end{array}$ & 2 & 1 \\
\hline Vinorelbine $20 \mathrm{mg}$ & 0 & 2 \\
\hline No chemotherapy & 2 & 0 \\
\hline \multicolumn{3}{|l|}{ Number of cycles given } \\
\hline 1 & 1 & 2 \\
\hline 2 & 5 & 2 \\
\hline 3 & 2 & 2 \\
\hline 4 & 4 & 3 \\
\hline 5 & 42 & 11 \\
\hline 6 & 17 & 4 \\
\hline $10^{\mathrm{b}}$ & 1 & 0 \\
\hline No data & 3 & 1 \\
\hline \multicolumn{3}{|l|}{ Radiotherapy pelvis } \\
\hline $\begin{array}{l}\text { Total dose: mean } \\
\quad \text { (range; Gy) }\end{array}$ & $44.6(39.6-46.2)$ & $44.6(39.6-45.0)$ \\
\hline$<45 \mathrm{~Gy}$ & 9 & 2 \\
\hline $45 \mathrm{~Gy}$ & 67 & 23 \\
\hline$>45 \mathrm{~Gy}$ & 1 & 0 \\
\hline \multicolumn{3}{|c|}{ Radiotherapy of paraaortic nodes performed } \\
\hline Yes & 20 & 5 \\
\hline No & 57 & 20 \\
\hline
\end{tabular}

Table 1 continued

\begin{tabular}{lll}
\hline & $\begin{array}{l}\text { Non-elderly } \\
\text { patients } \\
(n=77)\end{array}$ & $\begin{array}{l}\text { Elderly patients } \\
(n=25)\end{array}$ \\
\hline $\begin{array}{l}\text { Brachytherapy performed } \\
\text { Yes }\end{array}$ & 51 & 16 \\
No & 26 & 9 \\
Pelvic recurrence & \\
No & $56(75 \%)$ & $15(60 \%)$ \\
Yes & $19(25 \%)$ & $10(40 \%)$ \\
\hline
\end{tabular}

a All drug doses per sqm BSA

${ }^{\mathrm{b}}$ On an individual base due to persistent disease

c Brachytherapy performed by 192-Iridium high-dose rate intracavitary radiation

d 2 cases lost (non-elderly), in 4 no data upon dose reduction (3 nonelderly, 1 elderly)

NS not significant

\section{Acute toxicity}

All patients were assessable for acute toxicity. In Table 2, all adverse effects in correlation to age cohorts are shown. The majority of adverse reactions encountered were mild (grades 1 or 2).Of 102 patients, 38 experienced any kind of grade 3 or 4 toxicity, mainly hematologic and gastrointestinal. The most frequent adverse effects observed were hematologic. Any grade of thrombocytopenia occurred more frequently in the elderly ( 26 vs. $48 \%, p=0.004$ ). Grade 3 and 4 leukopenia, anemia, diarrhea and nausea were more frequent in the elderly (Table 2). All other acute morbidities listed occurred with comparable frequency in the two age groups. No toxic death (grade 5) occurred.

Chemotherapy, modification of treatment intensity

Two non-elderly were excluded from any chemotherapy due to severe co-morbidities and renal insufficiency. Data upon drug dose reduction performed were not fully retrievable in four (three non-elderly, one elderly) patients. From the remaining 96 patients, $56(60 \%)$ received the drug regimen without any modification. Forty $(40 \%)$ patients needed either any kind of drug dose reduction or an unscheduled treatment break due to acute adverse effects irrespective of the schedule applied. The need for reduced drug dose in any cycle due of the adverse effects did not differ between the age groups $(p=0.690) .16(22 \%)$ non-elderly and 6 (25\%) elderly needed a modification of the scheduled drug dose. However, the frequency of unscheduled breaks of their treatment due to acute morbidity was significantly age dependent $(p=0.002)$. 10 out of $77(13 \%)$ non-elderly 
Table 2 Incidence of the highest grade of acute adverse effects (CTC-Classification [NIH]) observed during treatment according to age a $A L T$ alaninaminotransferase, above upper normal limit

b AST aspartataminotransferase, above upper normal limit

\begin{tabular}{|c|c|c|c|c|}
\hline \multirow[t]{2}{*}{ Effect } & \multicolumn{2}{|c|}{$\begin{array}{l}\text { Highest grade of adverse } \\
\text { effect non-elderly }(n=77)\end{array}$} & \multicolumn{2}{|c|}{$\begin{array}{l}\text { Highest grade of adverse } \\
\text { effect elderly }(n=25)\end{array}$} \\
\hline & $\begin{array}{l}1 / 2 \\
n(\%)\end{array}$ & $\begin{array}{l}3 / 4 \\
n(\%)\end{array}$ & $\begin{array}{l}1 / 2 \\
n(\%)\end{array}$ & $\begin{array}{l}3 / 4 \\
n(\%)\end{array}$ \\
\hline Leukopenia & $43(56)$ & $21(27)$ & $10(40)$ & $8(32)$ \\
\hline Anemia & $29(38)$ & $2(3)$ & $7(28)$ & $1(4)$ \\
\hline Diarrhea & $26(34)$ & $1(1)$ & $8(32)$ & $1(4)$ \\
\hline Nausea & $24(31)$ & $1(1)$ & $10(40)$ & $1(4)$ \\
\hline Thrombopenia & $20(26)$ & 0 & $12(48)$ & 0 \\
\hline Skin hyperpigmentation & $11(14)$ & 0 & $2(8)$ & 0 \\
\hline Epitheliolysis & $10(13)$ & 0 & $2(8)$ & 0 \\
\hline Erythema & $8(10)$ & 0 & $5(20)$ & 0 \\
\hline $\begin{array}{l}\text { Creatinine clearance below } \\
\text { lower normal limit }\end{array}$ & $7(9)$ & 0 & $1(4)$ & 0 \\
\hline Emesis & $7(9)$ & 0 & $4(16)$ & 0 \\
\hline Constipation & $5(6)$ & 0 & $1(4)$ & 0 \\
\hline ALT increase ${ }^{a}$ & $5(6)$ & 0 & 0 & 0 \\
\hline AST increase ${ }^{b}$ & $3(4)$ & 0 & 0 & 0 \\
\hline Hypocalcemia & $3(4)$ & 0 & $1(4)$ & 0 \\
\hline Loss of appetite & $3(4)$ & 0 & $1(4)$ & 0 \\
\hline Creatinine increase & $2(3)$ & 0 & 0 & 0 \\
\hline Cystitis & $2(3)$ & 0 & 0 & 0 \\
\hline Hypokalemia & $2(3)$ & 0 & 0 & 0 \\
\hline Pruritus & $2(3)$ & 0 & 0 & 0 \\
\hline Seizure & $1(1)$ & $1(1)$ & 0 & 0 \\
\hline Enteritis & $1(1)$ & 0 & 0 & 0 \\
\hline Fever & $1(1)$ & 0 & 0 & 0 \\
\hline Dizziness & 0 & 0 & $1(4)$ & 0 \\
\hline Smell alteration & 0 & 0 & $1(4)$ & 0 \\
\hline Taste alteration & 0 & 0 & $1(4)$ & 0 \\
\hline
\end{tabular}

patients and 11 out of $25(44 \%)$ of the elderly needed a treatment interruption.

Non-elderly (69) and elderly (21) patients scheduled for $40 \mathrm{mg} / \mathrm{sqm}$ BSA cis-platinum received a median cumulative dose of $190 \mathrm{mg} / \mathrm{sqm}$ BSA and $173 \mathrm{mg} / \mathrm{sqm} \mathrm{BSA}$, respectively. 42/69 non-elderly patients scheduled for $40 \mathrm{mg} / \mathrm{sqm}$ BSA cis-platinum received 5 cycles and 16 receive 6 cycles. Nine out of twenty-one non-elderly patients scheduled for $40 \mathrm{mg} / \mathrm{sqm}$ BSA cis-platinum received 5 cycles and 4 received 6 cycles (Fig. 1).

\section{Recurrence}

Pelvic recurrence and distant metastases were observed in 36 non-elderly (48\%) and 12 elderly patients (48\%). The frequency of relapses was not related with modification of the scheduled therapy (non-elderly: $p=0.152$; elderly: $p=0.428)$. Pelvic recurrence were observed in $19(25 \%)$ non-elderly and $10(40 \%)$ elderly patients. $8(32 \%)$ of the

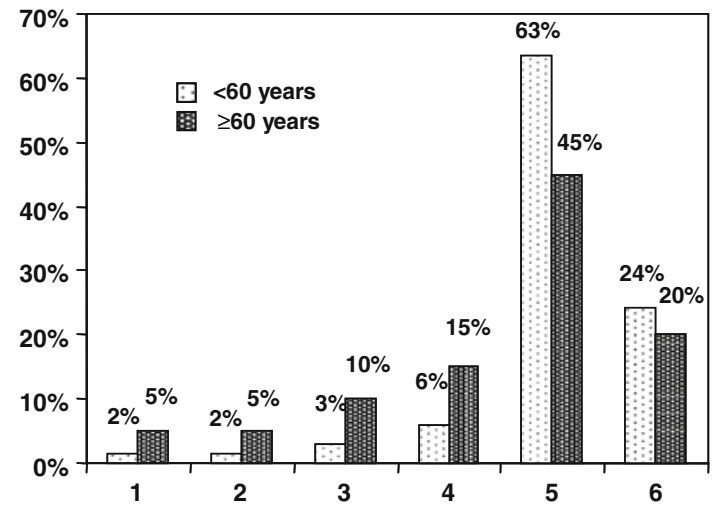

Fig. 1 Frequency of weekly cycles of cis-platinum, $40 \mathrm{mg} / \mathrm{sqm}$ BSA given in non-elderly and elderly patients

non-elderly patients and $5(50 \%)$ of the elderly patients needed a modification of the scheduled therapy ( $p=$ not significant). Pulmonary metastases were observed in six, 
bone metastases in five, hepatic metastases in three and lymph node metastasis in three non-elderly patients. In the elderly in two cases pulmonary metastases were encountered.

Overall and progression-free survival

Five-year overall survival for the entire group was $46 \pm 5 \%$ (median: 46 months) and has not been found influenced by age or treatment breaks (age: $p=0.882$; treatment breaks: $p=0.859$ ). The 5 -year overall survival rate is $47 \pm 6 \%$ for non-elderly patients and $45 \pm 10 \%$ for the elderly (Fig. 2). The 5-year overall survival rate is $39 \pm 11 \% / 51 \pm 6 \%$ $(p=0.759)$ in patients with/without treatment breaks. The median survival is $46 / 35$ months in patients with/without treatment breaks. Overall survival at 5 years however was influenced by the FIGO stage $(p=0.036)$. The 5 -year overall survival rate is $53 \pm 6 \%$ for patients with FIGO I-II and $30 \pm 8 \%$ for patients with FIGO III-IV. The median survival is 32 months for the patients with FIGO III-IV.

5 -year progression-free survival for the entire group was $48 \pm 5 \%$, (median progression-free survival: 41 months). Also progression-free survival was found independent from age or treatment breaks (age: $p=0.963$; treatment breaks: $p=0.492$ ). The 5-year progression-free survival rate is $49 \pm 6 \%$ for non-elderly patients and $47 \pm 11 \%$ for elderly (Fig. 3). Median progression-free survival time is 43 versus 41 months in the non-elderly and the elderly, respectively. Patients with/without treatment breaks had a 5-year progression-free survival rate of $50 \pm 12 \% / 48 \pm 6 \%$. The 5 -year progression-free survival depends on FIGO stage $(p=0.036)$. The 5 -year progression-free survival rate is

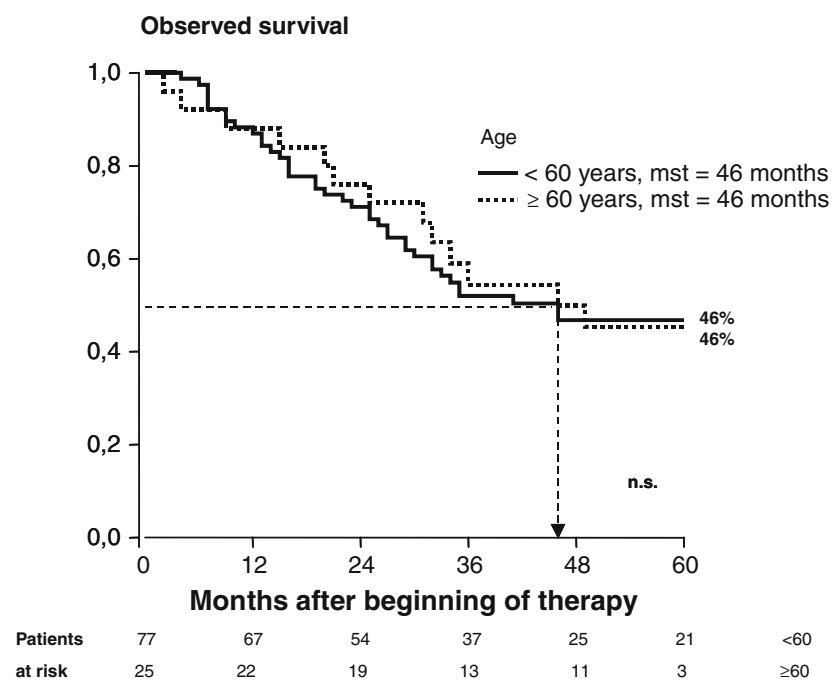

mst $=$ median survial time $;<60=<60$ years, $\geq 60=\geq 60$ years

Fig. 2 Overall survival according to age at diagnosis in 102 patients with high-risk cervical cancer
Progression free survival

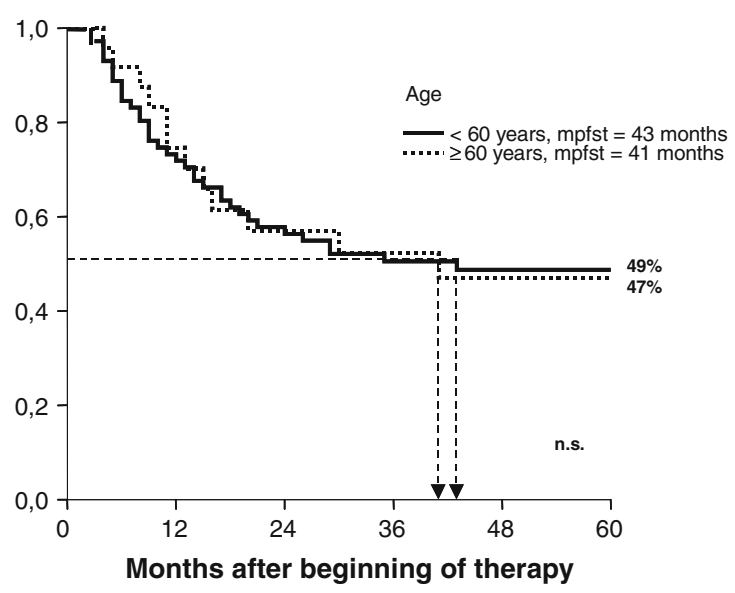

$\begin{array}{lllrrrrr}\text { Patients } & 71 & 52 & 41 & 32 & 23 & 19 & <60 \\ \text { at risk } & 24 & 18 & 13 & 10 & 9 & 3 & \geq 60\end{array}$

mpfst $=$ median progression free survial time; $<60=<60$ years, $\geq 60=\geq 60$ years

Fig. 3 Progression-free survival according to age at diagnosis in 102 patients with high-risk cervical cancer

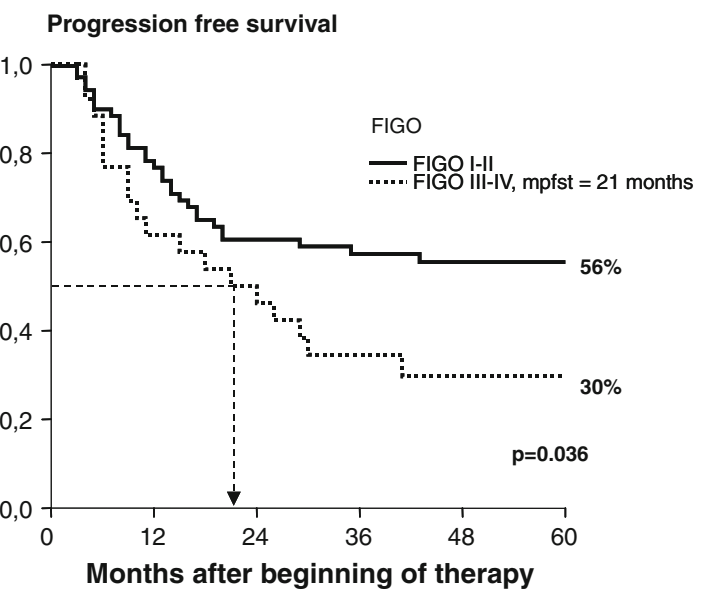

$\begin{array}{lccccccc}\text { Patients } & 69 & 54 & 42 & 34 & 26 & 19 & \text { FIGO I-II } \\ \text { at risk } & 26 & 16 & 13 & 8 & 6 & 3 & \text { FIGO III-IV } \\ \text { mpfst = median progression free survial time; I-II = FIGO I-II, III-IV = FIGO III-IV }\end{array}$

Fig. 4 Progression-free survival according to FIGO stage at diagnosis in 102 patients with high-risk cervical cancer

$56 \pm 6 \%$ for patients with FIGO I-II and $30 \pm 9 \%$ for patients with FIGO III-IV (Fig. 4). The median progression-free survival is 21 months for the patients with FIGO III-IV.

\section{Discussion}

In the eastern part of Germany including the catchment area of this study, the epidemiological data show an incidence of cervical cancer with a high peak at 50.5 years and a second lower peak beyond 65 years. Many factors may have 
contributed to halved mortality observed between 1990 and 2005 (Stabenow et al. 2009). However, age has been identified as adverse prognostic factor in some studies (Brun et al. 2003, Croker et al. 2009; Wright et al. 2005), while in others not (Kunos et al. 2009; Metindir and Bilir 2007; Mitchell et al. 1998). If age is associated with worse prognosis due to different reasons, this would also change the observed evolution of mortality in a senescent population in the future. To continue the downward trend of cervical cancer mortality it will be crucial to improve outcome in the elderly. The results obtained shed light upon the practicability of an intensified treatment protocol also in the aged.

One explanation for unfavorable prognosis might be the elderly present with more advanced tumor stages as shown in previous studies (Brun et al. 2003). In the present study, however, the elderly did neither present with more advanced tumor stages nor with a higher rate of prognostically unfavorable adenocarcinoma which agrees with other reports (Goodheart et al. 2008; Mitchell et al. 1998). In this series, both age cohorts revealed node metastases in 50-60\% of all patients which is much higher than in other reports (Rose et al. 1999; Eifel et al. 2004) and will have contributed to the unfavorable overall survival. Another reason for decreasing survival with increasing age may be an impaired tolerance of aggressive multimodal treatment protocols (Kirwan et al. 2003) or omission of simultaneously given chemotherapy at all in a subset of the elderly deemed not eligible for chemoradiation (Goodheart et al. 2008). However, radiotherapy alone neither postoperatively nor as the primary therapy is any longer the standard of care for highrisk cervical cancer even when very sophisticated techniques are used (Dimopoulos et al. 2009).

The accrual period of this analysis begun purposely after the NCI clinical announcement has recommended integrating cis-platinum to a standard radiotherapy protocol (McNeil 1999). Since the end of 1999 weekly cis-platinum doses given concurrently to pelvic radiotherapy has been adopted for postoperatively treated high-risk patients, for patients with inoperable tumors and for those medically unfit for surgery of the primary. Especially cisplatinum compounds given concomitantly to postoperative radiation therapy will lead to an increased overall survival as shown in a metaanalysis (Green et al. 2001; Anonymus 2008). At the same time, laparoscopic pelvic and retroperitoneal lymphadenectomy had become an internal standard procedure for all patients with cervical cancer irrespective of the management of the primary and of the patients' age at our Department of Gynecology and Obstetrics (Altgassen et al. 2000). Both changes in clinical practice undoubtedly alter the profile and intensity of treatment-induced morbidity.

In this study, all patients with an ECOG index of 0-2 irrespective of age were scheduled for the same chemother- apy protocol. Therefore, we should be able to determine age-related differences in terms of treatment-related morbidity and its impact on feasibility of an aggressive radiochemotherapy protocol in the elderly. Radiotherapy and cis-platinum have an overlapping toxicity profile. Therefore, when chemotherapy is given to pelvic radiotherapy their specific toxicities will add or potentate in part. Drugrelated hematological morbidity will be aggravated by exposure of large volumes of bone marrow to X-rays particularly in pelvic radiotherapy leading to acute and even chronic hematopoietic depression. In this series, severe acute hematological morbidity was found more frequent in the elderly, which is a common finding with different drug regimes (Peters et al. 2000). This has been anticipated since bone marrow is less likely to recover from chemotherapy in the elderly. New radiotherapy technologies may not alter this toxicity, since intensity-modulated radiotherapy is optimized essentially for small bowel and not pelvic bones' sparing.

Although age is not a contraindication for radical radiotherapy for pelvic tumors (Dimopoulos et al. 2009; Pignon et al. 1997) bowel derived toxicity has been reported to increase with age in patients receiving radiotherapy alone (Huscher et al. 2009). When pelvic irradiation given alone to patients 70 years and over in $29 \%$ unscheduled treatment breaks were unavoidable due to acute toxicity (Lindegaard et al. 2000). Similar figures have been reported after external radiation combined with three fractions of low-dose-rate brachytherapy without concurrent chemotherapy (Sakurai et al. 2000; Minagawa et al. 1997). These figures were comparable to the presented data despite chemotherapy have been added. This might be attributed to irradiation technology, e.g. using high-energy photons (15 MV) substituting cobalt X-rays and probably more important the use of bowel sparing intensity modulated radiotherapy.

Close clinical and laboratory monitoring has proven essential when aggressive radiochemotherapy is given to the elderly. We observed significantly more treatment breaks due to acute morbidity breaks which may be attributed to not sorting out patients deemed not suitable for this intensive protocol before start. Others found no differences in complication rates between younger and older women but emphasize not all of the elderly have been treated by chemotherapy (Goodheart et al. 2008). However, similar to our findings in the elderly drug dose reduction during radiochemotherapy was not more frequent compared to younger patients treated with the same protocol (Kunos et al. 2009).

In conclusion, our data demonstrate elderly patients tolerate radiochemotherapy worse than younger patients. Elderly may be treated safely with aggressive multimodal protocols but demand for more intensive monitoring in order to change treatment protocol early due to acute 
morbidity. However, significantly more treatment breaks do not compromise overall and progression-free survival.

\section{Conflict of interest statement None.}

Open Access This article is distributed under the terms of the Creative Commons Attribution Noncommercial License which permits any noncommercial use, distribution, and reproduction in any medium, provided the original author(s) and source are credited.

\section{References}

Ahamad A, D'Souza W, Salehpour M, Iyer R, Tucker SL, Jhingran A, Eifel PJ (2005) Intensity_modulated radiation therapy after hysterectomy: comparison with conventional treatment and sensitivity of the normal-tissue-sparing effect to margin size. Int J Radiat Oncol Biol Phys 62:1117-1124

Altgassen C, Possover M, Krause N, Plaul K, Michels W, Schneider A (2000) Establishing a new technique of laparoscopic pelvic and para-aortic lymphadenectomy. Obstet Gynecol 95:348-352

Anonymus (2008) Reducing uncertainties about the effects of chemoradiotherapy for cervical cancer: a ystematic review and metaanalysis of individual patient data from 18 randomized trials. J Clin Oncol 26:5802-5812

Brun JL, Stoven-Camou D, Trouette R, Lopez M, Chene G, Hocké C (2003) Survival and prognosis of women with invasive cervical cancer according to age. Gynecol Oncol 91:395-401

Croker AL, Eggleston KS, Du XL, Ramondetta L (2009) Ethnic disparities in cervical cancer survival among medicare eligible women in a multiethnic population. Int J Gynecol Cancer 19:13-20

Dimopoulos JCA, Schirl G, Baldinger A, Helbich TH, Pötter R (2009) MRI assessment of cervical cancer for adaptive radiotherapy. Strahlenther Onkol 185:282-287

Eifel PJ, Winter K, Morris M, Levenback C, Grigsby PW, Cooper J, Rotman M, Gershenson D, Mutch DG (2004) Pelvic irradiation with concurrent chemotherapy versus pelvic and para-aortic irradiation for high-risk cervical cancer: an update of radiation therapy oncology group trial (RTOG) 90-01. J Clin Oncol 22:872-880. doi: 10.1200/JCO.2004.07.197

Füller J, Guderian D, Köhler C, Schneider A, Wendt TG (2008) Lymph edema of the lower extremities after lymphadenectomy and radiotherapy for cervical cancer. Strahlenther Onkol 184:206-211

Goodheart M, Jacobson G, Smith BJ, Zhou L (2008) Chemoradiation for invasive cervical cancer in elderly patients: outcomes and morbidity. Int J Gynecol Cancer 18:95-103

Green JA, Kirwan JM, Tierney JF, Symonds P, Fresco L, Collingwood M, Williams CJ (2001) Systematic review and meta-analysis of randomised trials of concomitant chemotherapy and radiotherapy for cancer of the uterine cervix: better survival and reduced distant recurrence rate. Lancet 358:781-786

Huscher A, Bignardi M, Magri E, Vitali E, Pasinetti N, Costa L, Frata P, Magrini SM (2009) Determinants of small bowel toxicity in postoperative pelvic irradiation for gynaecological malignancies. Anticancer Res 11:4821-4826

Kirwan JM, Symonds P, Green JA, Tierney J, Collingwood M, Williams CJ (2003) A systematic review of acute and late toxicity of concomitant chemo-radiation for cervical cancer [review]. Radiother Oncol 68:217-226
Köhler C, Klemm P, Schau A, Possover M, Krause N, Tozzi R, Schneider A (2004) Introduction of and/or paraaortic transperitoneal lymphadenectomies. Gynecol Oncol 95:52-61

Kunos C, Tian C, Waggoner S, Rose PG, Lanciano R (2009) Retrospective analysis of concomitant cisplatin during radiation in patients aged 55 years or older for treatment of advanced cervical cancer: a gynecologic oncology group study. Int J Gynecol Cancer 19:1258-1263

Lindegaard JC, Thranov IR, Engelholm SA (2000) Radiotherapy in the management of cervical cancer in elderly patients. Radiother Oncol 56:9-15

McNeil C (1999) New standard of care for cervical cancer sets stage for next questions. J Natl Cancer Inst 91:500-513

Metindir J, Bilir G (2007) Prognostic factors affecting disease-free survival in early-stage cervical cancer patients undergoing radical hysterectomy and pelvic-araaortic lymphadenectomy. Eur J Gynaecol Oncol 28:28-32

Minagawa Y, Kigawa J, Itamochi H, Terakawa N (1997) The outcome of radiation therapy in elderly patients with advanced cervical cancer. Int J Gynaecol Obstet 58:305-309

Mitchell PA, Waggoner S, Rotmensch J, Mundt AJ (1998) Cervical cancer in the elderly treated with radiation therapy. Gynecol Oncol 71:291-298

Morris M, Eifel PJ, Lu J, Grigsby PW, Levenback C, Stevens RE, Rotman M, Gershenson DM, Mutch DG (1999) Pelvic radiation with concurrent chemotherapy compared with pelvic and paraaortic radiation for high-risk cervical cancer. $\mathrm{N}$ Engl $\mathrm{J}$ Med 340:1137-1143

NIH (1998) Common terminology criteria for adverse events v 2.0. National Institutes of Health, Bethesda

Peters WA III, Liu PY, Barrett RJ II, Stock RJ, Monk BJ, Berek JS, Souhami L, Grigsby P, Gordon W Jr, Alberts DS (2000) Concurrent chemotherapy and pelvic radiation therapy compared with pelvic radiation therapy alone as adjuvant therapy after radical surgery in high-risk early-stage cancer of the cervix. J Clin Oncol 18:1606-1613

Pignon T, Horiot JC, Bolla M, van Poppel H, Bartelink H, Roelofsen F, Pene F, Garard A, Einhorn N, Nguyen TD, Vanglabbeke M, Scalliet $\mathrm{P}$ (1997) Age is not a limiting factor for radical radiotherapy in pelvic malignancies. Radiother Oncol 42:107-120

Rose P, Bundy B, Watkins E, Thigpen JT, Deppe G, Maiman M, Clarke-Pearson DL, Insalaco S (1999) Concurrent cisplatin-based radiotherapy and chemotherapy for locally advanced cervical cancer. N Engl J Med 340:1144-1153

Sakurai H, Mitsuhashi N, Takahashi M, Yamakawa M, Akimoto T, Hayakawa K, Niibe H (2000) Radiation therapy for elderly patient with squamous cell carcinoma of the uterine cervix. Gynecol Oncol 77:116-120

Stabenow R, Streller B, Wilsdorf-Köhler H, Eisinger B (2009) Gemeinsames Krebsregister. Krebsinzidenz und Krebsmortalität 2005/ 2006. http://www.berlin.de/gkr/publikationen/jahresberichte/

Vandecasteele K, De Neve W, De Gersem W, Delrue L, Paelinck L, Makar A, Fonteyne V, De Wagter C, Villeirs G, De Meerleeer G (2009) Intensity moduated arc therapy with simultaneous integrated boost in the treatemtn of primary irresectable cervical cancer. Treatment planning, quality control and clinical implementation. Strahlenther Onkol 185:799-807

Wright JD, Gibb RK, Geevarghese S, Powell MA, Herzog TJ, Mutch DG, Grigsby PW, Gao F, Trinkaus KM, Rader JS (2005) Cervical carcinoma in the elderly. An analysis of patterns of care and outcome. Cancer 103:85-91 